\title{
Connecting concepts about current to quantitative circuit problems
}

\author{
Stephen Kanim
}

Department of Physics, New Mexico State University, Las Cruces, New Mexico 88003

This paper describes portions of an ongoing investigation into the relationship between conceptual knowledge and problem-solving ability in physics. ${ }^{1}$ To what degree do students apply conceptual knowledge to the solution of traditional examination and end-of-chapter physics problems? Are there instructional strategies that can facilitate this application? Finally, does an increased emphasis on developing conceptual understanding of the material underlying these problems have any impact on subsequent coursework?

\section{Context for the Investigation}

The primary context for this investigation was the electricity and magnetism portion of the introductory calculus-based physics course at the University of Washington. Most of the students enrolled in this course are engineering majors. Fewer than 5\% are physics majors. Electricity and Magnetism, the second quarter of a three-quarter sequence, is preceded by a quarter of Mechanics and followed by a quarter of Waves and Optics. There are three fifty-minute lectures each week, a required three-hour laboratory, and a fifty-minute tutorial. In tutorial, students work in small groups on exercises intended to address common conceptual difficulties. These exercises, from Tutorials in Introductory Physics, ${ }^{2}$ have been designed on the basis of research into student understanding of specific topics in the introductory course. The tutorials focus on promoting conceptual understanding, on developing models to account for observations, and on strengthening students' ability to reason about physical phenomena on the basis of available evidence: The tutorials do not emphasize quantitative problem solving.

In the electric circuits tutorial, students develop a qualitative model that allows them to reason about current in electric circuits. After participating in this tutorial, students are much more successful at reasoning qualitatively about current in electric circuits. ${ }^{3}$

To test whether students who demonstrate that they have developed a conceptual model for electric circuits are better able to solve quantitative circuits problems, two questions were asked on a midterm examination in two different sections (with different instructors) of an Electricity and Magnetism class at the University of Washington. Students had completed all instruction in electric circuits at the time of the midterm examination, and had also completed a laboratory on electric circuits and two tutorials on electric circuits. As part of a series of questions on the first page of the examination, students were asked to rank the currents through the four bulbs of the circuit shown in Figure 1.

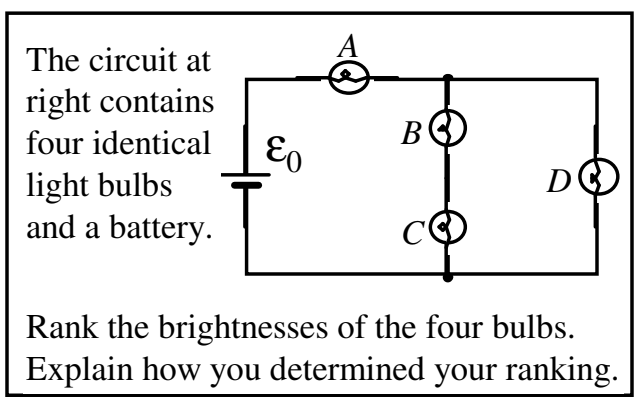

Figure 1. Paired circuits question -qualitative.

A correct response requires that students recognize that the current will split at the junction after bulb $\mathrm{A}$ and that there will be more current in the branch 
with the least resistance (the branch containing bulb D). Students must also relate the brightness of identical bulbs to the current through them. Below is a correct response from a student:

" $A>D>B=C$. $A$ is in series with the power supply but $D$ is in parallel with $B$ $\& C$. Since the current splits it is higher for $A$ than the rest. $D$ has less resistance than $B$ \& $C$ combined so more current will flow through it."

This is an example of reasoning based on the model developed in the tutorial on electric circuits.

As part of a series of questions on the third page of the same examination, students were asked to express the current through each resistor in terms of the current through the battery for the circuit shown in Figure 2.

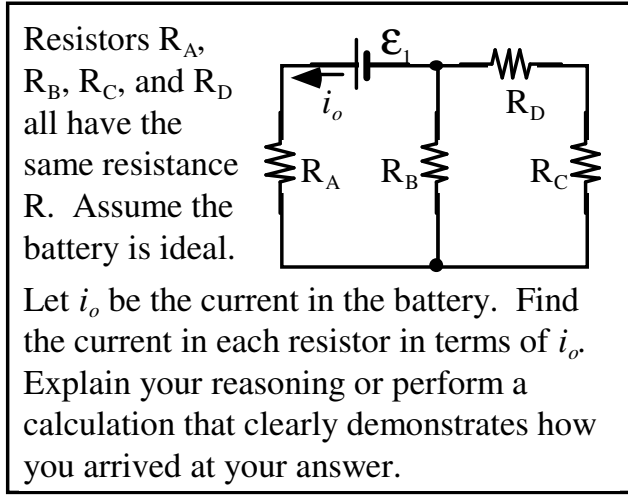

Figure 2. Paired circuits question -quantitative.

This second question requires that students extend the model developed in tutorial to a quantitative analysis. The following student quote is an example of this extension:

" $i_{A}=i_{o} \quad i_{B}=2 / 3 i_{o} \quad i_{C}=i_{D}=1 / 3 i_{o}$. The current $i_{o}$ is the same thru $R_{A}$, splits and is greater thru $R_{B}$ than thru $R_{D}$ and $R_{C}$. $2 x$ the current goes thru $R_{B}$ because it has $1 / 2$ the resistance."
The results from the two sections were similar and have been combined in Figure 3.

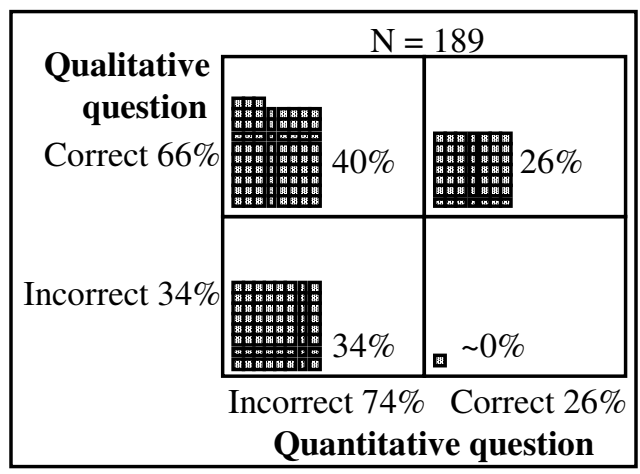

Figure 3. Correlation of performance on qualitative and quantitative questions -post tutorial.

Each shaded square represents a single student. One indication of the effectiveness of the tutorials is that twothirds were able to rank the bulb brightness with correct (although sometimes incomplete) reasoning. Only about one-quarter were able to answer the related quantitative question (upper right quadrant). Only one student who could not do the ranking task could calculate the current (lower right quadrant).

About $40 \%$ of the students were able to answer the qualitative question but were unable to answer the quantitative question (upper left quadrant).

This result is consistent with results reported by other researchers who have investigated student performance on quantitative physics questions after curriculum has been implemented that focuses on conceptual development. Even in cases where less time is spent on problem-solving as a result of modifications to the curriculum, students typically do slightly better on quantitative problems. 
Why only slightly better? A closer look at student solutions to the two questions suggests that many did not approach the quantitative question in the same manner in which they approached the qualitative question. A common pair of responses was a correct qualitative response to the bulb circuit (Figure 1) and an equation-driven response to the quantitative question (Figure 2) that seemed to have no direction and included no written description. This result was consistent with our analysis of interview responses that suggested that many students did not make a strong connection between the concepts developed in tutorials and the traditional problems they were being asked to solve in homework and on examinations.

We designed a series of homework worksheets intended to more strongly link concepts and traditional problems. These worksheets contained 'bridging exercises' that typically started with conceptual questions that were very similar to questions asked in the tutorials, and then progressed to more quantitative analysis of standard problems. Where possible, we based the questions that we asked on the worksheets on known conceptual and procedural difficulties. ${ }^{1}$

To measure the effectiveness of these exercises, we again asked the paired conceptual and traditional questions shown in Figures 1 and 2 in two sections of the course with different instructors. Results are shown in Figure 4: Over half of the students could now answer both questions correctly. Additionally, it appears that the additional reinforcement provided on the homework has served to improve students' conceptual understanding. About $84 \%$ were able to answer the qualitative question correctly.

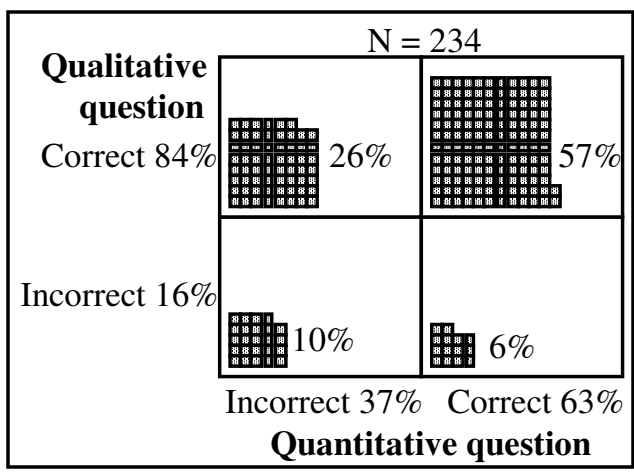

Figure 4. Correlation of performance on qualitative and quantitative questions post tutorial and 'bridging exercises'

The bridging exercises were not as successful for topics where students' conceptual difficulties are not as well understood. In some areas such as electrostatics, poor results after the homework worksheets led to additional research into students' conceptual difficulties. ${ }^{4}$ This research led in turn to modifications to the tutorials and to the bridging exercises.

Effect of research-based instruction on subsequent coursework.

Some of the introductory physics courses for engineers at New Mexico State University have in the past few years been conducted with an increased emphasis on conceptual understanding as part of the lecture. Homework assignments for these pilot courses have been modified to include bridging exercises. We have been monitoring the progress of students in these courses as they complete subsequent engineering coursework. Preliminary results are encouraging. For example, of the 29 students who completed an engineering statics course after completing a pilot mechanics course, 20 received A's. Only two students earned a lower grade in engineering statics than they did in their mechanics course. Nineteen of these twenty-nine students received 
higher grades in their engineering courses than they did in their physics course, even though the grade distributions were about the same. In contrast, of 202 students who completed engineering statics after completing a standard course in mechanics, 58 received a lower grade in statics than they did in their mechanics course, and 63 received a higher grade. These results suggest that the students in the modified courses with an increased emphasis on conceptual development were more successful in the subsequent course even though the evaluation of student performance in this subsequent course was based primarily on traditional quantitative problems.

We have been disappointed by the results of a similar look at student performance in an engineering electromagnetics course taken subsequent to a modified physics electricity and magnetism course. In these cases, students performed about as well in their engineering course as they did in their physics course. It is not clear why the same strategies that seem to be successful in mechanics are not successful in E\&M. There are two factors that may contribute to this difference. First, students in the modified E\&M course first took a standard course in mechanics. This first exposure could influence how students approach problems, and make it less likely that they will view concepts as an integral part of problem solving. Second, more research has been done on student difficulties in mechanics than in E\&M. It is possible that the homework exercises we have developed for mechanics are more effective because we understand more about the nature of student difficulties.

\section{Conclusion}

For topics where effective curricula are available to address student conceptual difficulties, the addition of an explicit link between concepts and traditional problems can serve both to reinforce concepts and to improve student quantitative problem-solving performance.

\section{Acknowledgements}

I would like to thank the many students who participated in the studies described in this paper. The assistance of all members of the Physics Education Group at the University of Washington is gratefully acknowledged, and in particular the substantial contributions made by Peter Shaffer and Lillian McDermott.

\section{References}

${ }^{1}$ S. Kanim, "An investigation of student difficulties in qualitative and quantitative problem solving: Examples from electric circuits and electrostatics," Ph.D. dissertation, Department of Physics, University of Washington (1999).

${ }^{2}$ L.C. McDermott, P. S. Shaffer, and the Physics Education Group at the University of Washington, Tutorials in Introductory Physics (Preliminary Edition), (Prentice Hall, Upper Saddle River, 1998).

${ }^{3}$ L.C. McDermott and P.S. Shaffer, "Research as a guide for curriculum development: An example from introductory electricity, Part I: Investigation of student understanding," American Journal of Physics 60(11), 994-1002 (1992).

${ }^{4}$ S. Kanim, "Connecting Concepts to Problem Solving," in these conference proceedings. 